\title{
SETS OF INTEGERS AVOIDING CONGRUENT SUBSETS
}

\author{
RAFAEL TESORO
}

\begin{abstract}
We study the sets of integers $A$ that avoid any arrangement of $g$ congruent $h$-subsets (the $C_{h}[g]$ sets, for short), as well as the variant in which the $h$-subsets are pairwise disjoint (the weak- $C_{h}[g]$ sets). For $h=g=2$ these are the Sidon sets and the weak-Sidon sets respectively.

We refine and improve upon several results by Erdős and Harzheim. For finite
\end{abstract} sets on the one hand we prove the upper bound

$$
|A| \leq(g-1)^{1 / h} n^{1-\frac{1}{h}}+O\left(n^{\frac{1}{2}-\frac{1}{2 h}}\right),
$$

for any $C_{h}[g]$ set $A \subset[1, n]$. On the other hand we prove that there exists a weak- $C_{h}[g]$ subset $A \subset[1, n]$ such that

$$
|A| \gg n^{\left(1-\frac{1}{h}\right)\left(1-\frac{1}{g}\right)\left(1+\frac{1}{h g-1}\right)} .
$$

For any infinite $C_{h}[g]$ sequence $A$ we prove that

$$
A(x) \ll x^{1-1 / h}(\log x)^{-1 / h}
$$

for infinitely many integers $x$.

We conclude stating several related open problems.

\section{INTRODUCTION}

We say that a set of integers $A$ is a $C_{h}[g]$ set if for any set $X$ of $h$ elements there do not exist distinct integers $k_{1}, \cdots, k_{g}$ such that $X+k_{1}, \cdots, X+k_{g} \subset A$. We write weak- $C_{h}[g]$ when the condition that the sets $X+k_{i}$ are pairwise disjoint is added. This generalization of Sidon sets was introduced by Erdös and Harzheim in [5].

Erdős and Harzheim used the term " $B_{g h}$-sequence". We prefer to write $C_{h}[g]$ (with $C$ as in "congruent") aiming to prevent confusion with the established convention of using $B_{h}[g]$ for a different generalization of Sidon sets. Our notation also allows to state in a concise way some symmetries such us the fact that any $C_{h}[g]$ set is also a $C_{g}[h]$ set, and vice versa. Indeed if $X_{1}, \cdots, X_{g} \subset A$ are congruent $h$-sets, for $\nu=1, \cdots, h$ let $Y_{\nu}$ be the set of the $\nu^{\text {th }}$ elements of the sets $X_{i}$. Then $Y_{1}, \cdots, Y_{h}$ are congruent $g$-subsets of $A$.

Date: $24 / 06 / 2013$.

I am grateful to Javier Cilleruelo for his suggestions and comments that improved the content of this paper. 
In the sequel we will assume $g \geq h \geq 2$. We will also use the following standard notation:

- $f(n) \ll g(n)$ or $f(n)=O(g(n))$ means that there exists $C>0$ such that $f(n)<C g(n)$ for all $n$.

- $f(x)=o(g(x))$ means that $f(x) / g(x) \rightarrow 0$ as $x \rightarrow \infty$.

- $f(x) \sim g(x)$ means that $f(x) / g(x) \rightarrow 1$ as $x \rightarrow \infty$.

Our first result is the following.

Theorem 1. If $A \subset[1, n]$ is a $C_{h}[g]$ set, with $g \geq h \geq 2$, then

$$
|A| \leq(g-1)^{1 / h} n^{1-\frac{1}{h}}+O\left(n^{\frac{1}{2}-\frac{1}{2 h}}\right) .
$$

This theorem is a refinement of the estimate $|A| \ll n^{1-1 / h}$ proved by Erdös and Harzheim. We remark that $C_{2}[2]$ sets are just Sidon sets and Theorem 1 recovers the well known upper bound for the size of Sidon sets in $\{1, \cdots, n\}$ obtained by Erdös and Turan [6]. In general $C_{2}[g]$ sets are those sets $A$ such that each difference $a-a^{\prime}$ appears at most $g-1$ times and Theorem 1 recovers Corollary 2.1 in [4].

In the opposite direction, Erdős and Harzheim proved that

"if $\alpha$ satisfies $0<\alpha<\left(1-\frac{1}{h}\right)\left(1-\frac{1}{g}\right)$, then for all sufficiently large natural numbers $n$ there exists a subset of $\{1, \cdots, n\}$ which has at least $n^{\alpha}$ elements but no $g$ disjoint congruent h-element subsets."

Erdős and Harzheim added the restriction that the sets are disjoint because it simplifies the proof. We do the same but obtain a better lower bound. It seems that the same lower bound should hold for $C_{h}[g]$ sets but we have not found a proof.

Theorem 2. There exists a weak- $C_{h}[g]$ subset $A$ of $[1, n]$ such that

$$
|A| \gg n^{\left(1-\frac{1}{h}\right)\left(1-\frac{1}{g}\right)\left(1+\frac{1}{h g-1}\right)} \text {. }
$$

It should be noted that for $h$ fixed, Theorem 2 gives $|A| \gg n^{1-\frac{1}{h}-\epsilon}$ for $g$ sufficiently large, being the lower bound close to the exponent given in Theorem 1. For small values of $g$, Theorem 2 is not so strong. Indeed there are algebraic constructions of Sidon sets (which are obviously $C_{h}[g]$ sets) of size $|A| \sim n^{1 / 2}$, while the exponent in Theorem 2 is not greater than $1 / 2$ for $h=2$ and for $(h, g)=(3,3)$. Theorem 2 gives non trivial lower bounds in any other case.

Theorem 1 and what is known about Sidon sets give the estimates

$$
n^{1 / 2}(1+o(1))<|A|<2^{1 / 3} n^{2 / 3}(1+o(1))
$$

for a $C_{3}[3]$ set $A \subset\{1, \ldots, n\}$ of maximum cardinality. Probably there exists an algebraic construction of a $C_{3}[3]$ set beating the exponent in the lower bound but we have not found it. 
We prove Theorem 2 using the probabilistic method combined with the deletion technique. A random finite set, say $S$, may not be weak- $C_{h}[g]$ but have some "blemishes", that is to say, some arrangements of $g$ congruent disjoint $h$-sets occur inside $S$. Blemishes are more likely the larger is $S$. We tune the construction so that the size of the random set moderately exceeds the threshold for the weak- $C_{h}[g]$ property. Then we can prune all the blemishes from $S$ obtaining a true weak- $C_{h}[g]$ set, while the size is roughly preserved. These ideas have appeared before in the literature, see for example [1, §3], [8], and [3].

Erdős and Harzheim also proved that for any $C_{h}[g]$ infinite sequence $A$ we have

$$
\liminf _{x \rightarrow \infty} \frac{A(x)}{x^{1-1 / h}}=0 .
$$

We refine this result as follows.

Theorem 3. If $A$ is an infinite $C_{h}[g]$ sequence then

$$
\liminf _{x \rightarrow \infty} A(x) \cdot \frac{(x \log x)^{1 / h}}{x} \ll 1,
$$

where the implicit constant depends on $g$ and $h$.

Theorem 3 was proved by Erdős [7] when $h=g=2$ (infinite Sidon sequences).

\section{Finite $C_{h}[g]$ SETS}

We start recalling a Theorem, which is a consequence of the Jensen's inequality, and that will be used next in the proof of Theorem 1.

Theorem 4 (Overlapping theorem [2]). Let $(\Omega, \mathcal{A}, \mathbb{P})$ be a probability space and let $\left\{E_{j}\right\}_{j=1}^{k}$ denote a family of events. Write

$$
\sigma_{m}:=\sum_{1 \leq j_{1}<\cdots<j_{m} \leq k} \mathbb{P}\left(E_{j_{1}} \cap \cdots \cap E_{j_{m}}\right), \quad(m \geq 1) .
$$

Then we have

$$
\sigma_{m} \geq\left(\begin{array}{l}
\sigma_{1} \\
m
\end{array}\right)=\frac{\sigma_{1}\left(\sigma_{1}-1\right) \cdots\left(\sigma_{1}-(m-1)\right)}{m !} .
$$

2.1. Proof of Theorem 1. Let $A$ be a $C_{h}[g]$ set and let $B$ be any subset of integers within $[1, n]$ of size at least $h$. Let $\mathbb{Y}$ be a random variable with range the positive integers and law

$$
\mathbb{P}(\mathbb{Y}=m)= \begin{cases}\frac{1}{|A+B|} & \text { if } m \in A+B \\ 0 & \text { otherwise }\end{cases}
$$


For every $b \in B$ we define the event $E_{b}=\{\omega \in \Omega: \mathbb{Y}(\omega) \in A+b\}$, that has probability $\mathbb{P}\left(E_{b}\right)=\sum_{a \in A} \mathbb{P}(\mathbb{Y}=a+b)=|A| /|A+B|$. We also write

$$
\sigma_{m}:=\sum_{\left\{b_{1}, \cdots, b_{m}\right\} \in\left(\begin{array}{c}
B \\
m
\end{array}\right)} \mathbb{P}\left(E_{b_{1}} \cap \cdots \cap E_{b_{m}}\right), \quad(m \geq 1) .
$$

In particular

$$
\sigma_{1}=\frac{|A||B|}{|A+B|} .
$$

Let $b_{1}>\cdots>b_{h}$ be $h$ fixed elements of $B$. We can write

$$
\begin{aligned}
\mathbb{P}\left(E_{b_{1}} \cap \cdots \cap E_{b_{h}}\right) & =\sum_{\left\{a_{1}, \cdots, a_{h}\right\} \in\left(\begin{array}{c}
A \\
h
\end{array}\right)} \mathbb{P}\left(\mathbb{Y}=a_{1}+b_{1}=a_{2}+b_{2} \cdots=a_{h}+b_{h}\right) \\
& =\sum_{a_{1}+\left\{0, b_{1}-b_{2}, b_{1}-b_{3}, \cdots, b_{1}-b_{h}\right\} \in\left(\begin{array}{c}
A \\
h
\end{array}\right)} \frac{1}{|A+B|},
\end{aligned}
$$

the sum extending to all $a_{1} \in A$ such that $a_{1}+\left\{0, b_{1}-b_{2}, b_{1}-b_{3}, \cdots, b_{1}-b_{h}\right\} \subset A$. These are congruent $h$-subsets of the $C_{h}[g]$ set $A$, thus

$$
\mathbb{P}\left(E_{b_{1}} \cap \cdots \cap E_{b_{h}}\right) \leq \frac{g-1}{|A+B|} .
$$

Now we use Theorem 4 to obtain

$$
\left(\begin{array}{c}
|B| \\
h
\end{array}\right) \frac{g-1}{|A+B|} \geq \sigma_{h} \geq \frac{\sigma_{1}\left(\sigma_{1}-1\right) \cdots\left(\sigma_{1}-h+1\right)}{h !} \geq \frac{\sigma_{1}}{h !}\left(\sigma_{1}-(h-1)\right)^{h-1},
$$

and so

which implies

$$
\frac{|B|^{h}}{h !} \frac{(g-1)}{|A+B|} \geq \frac{|A||B|}{h !|A+B|}\left(\frac{|A||B|}{|A+B|}-(h-1)\right)^{h-1},
$$

$$
|A|^{h /(h-1)} \leq|A+B|\left((g-1)^{1 /(h-1)}+\frac{(h-1)|A|^{1 /(h-1)}}{|B|}\right) .
$$

If we choose $B=[0, \ell]$, by the last inequality we have

$$
|A|^{h /(h-1)} \leq(n+\ell)\left((g-1)^{1 /(h-1)}+\frac{(h-1)|A|^{1 /(h-1)}}{\ell+1}\right) .
$$

We first take $\ell=n$ and use $|A| \leq n$ in the right side, getting $|A|^{h /(h-1)} \ll n \Longrightarrow$ $|A|^{1 /(h-1)} \ll n^{1 / h}$. Inserting this in the second member of (2.1) we obtain

$$
|A|^{h /(h-1)} \leq(g-1)^{1 /(h-1)} n+O(\ell)+O\left(\frac{n^{1+1 / h}}{\ell+1}\right)+O\left(n^{1 / h}\right) .
$$

To minimize this last upper bound we choose $\ell \asymp n^{1 / 2+1 / 2 h}$. Then we can write $|A|^{h /(h-1)} \leq(g-1)^{1 /(h-1)} n+O\left(n^{1 / 2+1 / 2 h}\right)=(g-1)^{1 /(h-1)} n\left(1+O\left(n^{1 / 2 h-1 / 2}\right)\right)$, 
which yields

$|A| \leq(g-1)^{1 / h} n^{1-1 / h}\left(1+O\left(n^{1 / 2 h-1 / 2}\right)\right)^{1-1 / h}=(g-1)^{1 / h} n^{1-1 / h}+O\left(n^{1 / 2-1 / 2 h)}\right)$.

2.2. Proof of Theorem 2. We say that $m \in S$ is $(h, g)$-bad (for $S$ ) if there exist $m_{1}<\cdots<m_{g-1}$, with $m_{i}<m$, and there exist $\ell_{1}<\ell_{2}<\cdots<\ell_{h-1}$ such that the sums $\left\{m_{1}, \cdots, m_{g-1}, m\right\}+\left\{0, \ell_{1}, \cdots, \ell_{h-1}\right\}$ are $g h$ distinct elements of $S$.

We define $S_{b a d}$ the set of $(h, g)$-bad elements for $S$. It is clear that for any set $S$, the set

$$
S_{C_{h}[g]}=S \backslash S_{b a d},
$$

is weak- $C_{h}[g]$ with cardinality $\left|S_{C_{h}[g]}\right|=|S|-\left|S_{\text {bad }}\right|$.

Define $p$ as the number such that $2 p n=n^{g+h-1}(2 p)^{h g}$. It is straightforward to check that

$$
n p=\frac{1}{2} n^{\left(1-\frac{1}{h}\right)\left(1-\frac{1}{g}\right)\left(1+\frac{1}{h g-1}\right)} .
$$

We will prove that except for finitely many $n$ there exist a set $S \subset[1, n]$ such that

$$
|S| \geq \frac{n p}{2} \quad \text { and } \quad\left|S_{b a d}\right| \leq \frac{n p}{4}
$$

Note that for such a set we have

$$
\left|S_{C_{h}[g]}\right|=|S|-\left|S_{b a d}\right|>\frac{n p}{4}=\frac{n^{\left(1-\frac{1}{h}\right)\left(1-\frac{1}{g}\right)\left(1+\frac{1}{h g-1}\right)}}{8},
$$

for all sufficiently large $n$ and $A=S_{C_{h}[g]}$ satisfies the conditions of Theorem 2 .

Indeed we will prove that with probability at least $1 / 4$, a random set $S$ in $[1, n]$ satisfies (2.3) if each element in $[1, n]$ is independently chosen to be in $S$ with probability $p$.

Next we obtain estimates for the random variables $|S|$ and $\left|S_{b a d}\right|$.

If $m$ is $(h, g)$-bad then the $g h$ sums $\left\{m_{1}, \cdots, m_{g-1}, m\right\}+\left\{0, \ell_{1}, \cdots, \ell_{h-1}\right\}$ are all distinct elements of $S$ and so

$$
\mathbb{P}\left(\left\{m_{1}, \ldots, m_{g-1}, m\right\}+\left\{0, \ell_{1}, \ldots, \ell_{h-1}\right\} \subset S\right)=p^{g h} .
$$

Hence

$$
\begin{gathered}
\mathbb{P}(m \text { is }(h, g) \text {-bad }) \leq \sum_{\substack{1 \leq m_{1}<\cdots<m_{g-1}<m \\
1 \leq \ell_{1}<\cdots<\ell_{h-1} \leq n}} p^{g h} \leq\left(\begin{array}{c}
m \\
g-1
\end{array}\right)\left(\begin{array}{c}
n \\
h-1
\end{array}\right) p^{g h}<n^{g+h-2} p^{g h} \\
\Longrightarrow \mathbb{E}\left(\left|S_{b a d}\right|\right) \leq \sum_{1 \leq m \leq n} \mathbb{P}(m \text { is } g \text {-bad }) \leq n^{g+h-1} p^{g h} .
\end{gathered}
$$


On the one hand by Markov's inequality we have

$$
\begin{aligned}
\mathbb{P}\left(\left|S_{b a d}\right|>\frac{n p}{4}\right) & =\mathbb{P}\left(\left|S_{b a d}\right|>\frac{n^{g+h-1}(2 p)^{g h}}{8}\right) \\
& =\mathbb{P}\left(\left|S_{b a d}\right|>2^{g h-3} n^{g+h-1} p^{g h}\right) \\
& \leq \mathbb{P}\left(\left|S_{b a d}\right|>2 \mathbb{E}\left(\left|S_{b a d}\right|\right)\right)<1 / 2 .
\end{aligned}
$$

On the other hand, using that $\mathbb{E}(|S|)=n p$ and $\operatorname{Var}(|S|)=n p(1-p)$ and applying Chebychev's inequality we have

$$
\begin{aligned}
\mathbb{P}\left(|S|<\frac{n p}{2}\right) & =\mathbb{P}\left(|S|<\frac{\mathbb{E}(|S|)}{2}\right)<\mathbb{P}\left(|S-\mathbb{E}(|S|)|>\frac{\mathbb{E}(|S|)}{2}\right) \\
& <\frac{4 \operatorname{Var}(|S|)}{(\mathbb{E}(|S|))^{2}}=\frac{4 n p(1-p)}{(p n)^{2}}<\frac{4}{p n}<\frac{1}{4}
\end{aligned}
$$

except for finitely many $n$. By (2.4) and (2.5) we have

$$
\mathbb{P}\left(|S| \geq n p / 2 \text { and }\left|S_{b a d}\right| \leq n p / 4\right) \geq 1-(1 / 2+1 / 4) \geq 1 / 4,
$$

as we wanted.

\section{Infinite $C_{h}[g]$ SEQUenCES}

3.1. Proof of Theorem 3. For a positive integer $N$, let $\left[0, N^{2}\right]$ denote all the positive integers less or equal to $N^{2}$. We divide $\left[0, N^{2}\right]$ into equally sized intervals

$$
I_{\nu}:=[(\nu-1) N, \nu N], \nu=1, \cdots, N .
$$

Let $\mathcal{C}$ denote the collection of all $h$-subsets of $\left[0, N^{2}\right]$ that are included in one of the intervals $I_{\nu}$ :

$$
\mathcal{C}:=\left\{C \in\left(\begin{array}{c}
{\left[0, N^{2}\right]} \\
h
\end{array}\right): C \subset I_{\nu} \text { for some } \nu\right\}
$$

We say that the sets in the collection $\mathcal{C}$ are "small" as their diameter is at most $N$. We classify the elements of $\mathcal{C}$ so that each class groups all the sets that are pairwise congruent. Each class $\alpha$ contains a set $C_{\alpha}$ that contains 0 , and the remaing $h-1$ elements of $C_{\alpha}$ can be chosen in $\left(\begin{array}{l}N-1 \\ h-1\end{array}\right)$ different ways; each of the choices determines a class different from the others. Then the number of classes is

$$
\left(\begin{array}{c}
N-1 \\
h-1
\end{array}\right)
$$

Let $A_{\nu}$ denote the size of $A \cap I_{\nu}$, we have $A_{\nu}=A(\nu N)-A((\nu-1) N)$, where $A(x):=|\{a \in A: a \leq x\}|$ is the counting function of the sequence.

One the one hand as $A$ is a $C_{h}[g]$ sequence then in every class of $\mathcal{C}$ there are at most $g-1$ subsets of $A$. Hence we have the following upper bound for the total 
number of "small" subsets of $A$ that belong to $\mathcal{C}$

$$
\sum_{\nu=1}^{N}\left(\begin{array}{c}
A_{\nu} \\
h
\end{array}\right) \leq\left(\begin{array}{c}
N-1 \\
h-1
\end{array}\right)(g-1) \ll N^{h-1} \quad(N \rightarrow \infty),
$$

Now we prove by induction in $h$ that

$$
\sum_{\nu=1}^{N} A_{\nu}^{h} \ll N^{h-1} \quad(N \rightarrow \infty)
$$

For $h=2$ we know by Theorem 1 that $A\left(N^{2}\right) \ll N$, so

$$
\sum_{\nu=1}^{N} A_{\nu}^{2}=2 \sum_{\nu=1}^{N}\left(\begin{array}{c}
A_{\nu} \\
2
\end{array}\right)+\sum_{\nu=1}^{N} A_{\nu} \ll N+A\left(N^{2}\right) \ll N
$$

If (3.1) holds for all exponents up to $h-1$, then

$$
\sum_{\nu=1}^{N} A_{\nu}^{h}=h ! \sum_{\nu=1}^{N}\left(\begin{array}{c}
A_{\nu} \\
h
\end{array}\right)+O\left(\sum_{\nu=1}^{N} A_{\nu}^{h-1}\right) \ll N^{h-1}+N^{h-2}, \quad(N \rightarrow \infty),
$$

thus it also holds for $h$. Using (3.1) and Hölder inequality we can write

$$
\begin{aligned}
\sum_{\nu=1}^{N} A_{\nu}\left(\frac{1}{\nu}\right)^{1-1 / h} & \leq\left(\sum_{\nu=1}^{N} A_{\nu}^{h}\right)^{1 / h}\left(\sum_{\nu=1}^{N} \frac{1}{\nu}\right)^{1-1 / h} \\
& \ll(N \log N)^{1-1 / h}, \quad(N \rightarrow \infty) .
\end{aligned}
$$

On the other hand as $\sum_{\nu \leq t} A_{\nu}=A(t N)$ and summing by parts

$$
\sum_{\nu=1}^{N} A_{\nu}\left(\frac{1}{\nu}\right)^{1-1 / h} \gg \int_{1}^{N} \frac{A(t N)}{t^{2-1 / h}} \mathrm{~d} t
$$

Let us write

$$
\tau(m):=\inf _{n \geq m} \frac{A(n)(\log n)^{1 / h}}{n^{1-1 / h}}
$$

For $N \geq m$ we have

$$
\int_{1}^{N} \frac{A(t N)}{t^{2-1 / h}} \mathrm{~d} t \gg \frac{\tau(m) N^{1-1 / h}}{(\log N)^{1 / h}} \int_{1}^{N} \frac{1}{t} \mathrm{~d} t \gg \tau(m)(N \log N)^{1-1 / h}
$$

and so

$$
\sum_{\nu=1}^{N} A_{\nu}\left(\frac{1}{\nu}\right)^{1-1 / h} \gg \tau(m)(N \log N)^{1-1 / h} .
$$

Inserting (3.2) we have $\lim _{m \rightarrow \infty} \tau(m) \ll 1$, that is what we wanted to prove. 


\section{Open pRoBlems}

Theorem 1 and what is known about Sidon sets give the estimates

$$
n^{1 / 2}(1+o(1))<|A|<2^{1 / 3} n^{2 / 3}(1+o(1))
$$

for a $C_{3}[3]$ set $A \subset\{1, \ldots, n\}$ of maximum cardinality.

Problem 1: Improve the bounds above.

Probably there exists an algebraic construction of a $C_{3}[3]$ set beating the exponent in the lower bound but we have not found it.

The analogous problem in two dimensions can be nicely illustrated.

Problem 2: What is the largest size of a set $A \in[1, n] \times[1, n]$ avoiding three translated triangles?

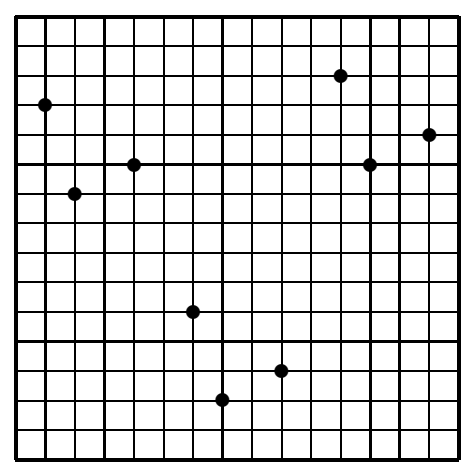

Our method would only give $n(1+o(1)) \leq|A| \ll n^{4 / 3}$.

Problem 3: Remove the condition weak in Theorem 2,

Problem 4: Construct infinite dense $C_{h}[g]$ sequences. At least with counting function $A(x) \gg x^{\left(1-\frac{1}{h}\right)\left(1-\frac{1}{g}\right)\left(1+\frac{1}{h g-1}\right)+o(1)}$.

We have found technical difficulties to deal with problems 3 and 4 . Erdős and Harzheim probably also found these difficulties, which would explain why they added the weak condition in his lower bound for the finite case and did not include the infinite case in their study.

\section{REFERENCES}

[1] Alon, N. and Spencer, J.: The Probabilistic Method, John Wiley \& Sons Inc., Third edition (2008).

[2] Cilleruelo, J. and Tenenbaum, G.: An Overlapping theorem with applications, Publicacions matemàtiques, Extra (2007).

[3] Cilleruelo, J.: Probabilistic constructions of $B_{2}[g]$ sequences, Acta Mathematica Sinica, English Series 26-7 (2010).

[4] Cilleruelo, J.: Sidon sets in $\mathbb{N}^{d}$, Journal of Combinatorial Theory, Series A 117 (2010). 
[5] Erdős, P. and Harzheim, E.: Congruent subsets of infinite sets of natural numbers, Journal fur die reine und angewandte Mathematik, 367 (1986).

[6] Erdős, P. and Turan, P.: On a problem of Sidon in additive number theory, and on some related problems, Journal of the London Mathematical Society, 16 (1941).

[7] Erdös, P.: Einige Bemerkungen zur Arbeit von A. Stöhr: "Gelöste und ungelöste Fragen über Basen der natürlichen Zahlenreihe" Journal für die reine und angewandte Mathematik, 197 (1957).

[8] Spencer, J. and Tetali P.: Sidon Sets with Small Gaps, Discrete Probability and Algorithms, The IMA Volumes in Mathematics and its Applications Volume 72, (1995), 103-109.

R. Tesoro: Departamento de Matemáticas, Universidad Autónoma de Madrid. 28049 MADRID, SPAIN

E-mail address: rafael.tesoro@estudiante.uam.es 